DOI: 10.17163/uni.n22.2015.01

\title{
Perspectivismo indígena
}

\section{Indigenous perspectivism}

\author{
Carlos Rojas Reyes \\ carlos.rojas@ucuenca.edu.ec
}

\section{Resumen}

El núcleo del perspectivismo, como afirmación clave de este trabajo, sostiene que los movimientos sociales construyen su propia realidad desde sus principios y programa, y que esta realidad y discursos resultantes atañen al conjunto de todo lo existente, desde la naturaleza hasta la tecnología pasando por los aspectos estrictamente sociales.

Sin embargo, este perspectivismo así visto constituiría una novedad bastante limitada y ya vista en otros procesos. Hay que añadir -lo que es fundamental, lo que es su esencia en el sentido estratégico de este términoes que la construcción de dicha perspectiva ontológica -y no solo cognoscitiva- se hace en base de "devorar" a la otra cultura, a la otra civilización, con la cual está confrontada.

Solo así tendrá pleno sentido los diversos componentes del perspectivismo que se analizan aquí, como estructuradores de los movimientos sociales: performatividad - que ya ha sido señalada antes-, vincularidad y aspectualidad.

De allí que la tesis central sea que cuando los indígenas viven y narran sus experiencias desde ellos mismos, no solo se están representando, sino que efectivamente crean sus culturas, permiten la emergencia y la continuidad de sus formas de vida. Y en este sentido avanzamos sobre las tesis de José Sánchez-Parga, para ir desde el reconocimiento a la constitución de las comunidades indígenas.

\section{Palabras claves}

Perspectivismo, indígena, performatividad, vincularidad, forma de vida.

\section{Abstract}

The core of perspectivism - as the key assertion underlying this work-claims that social movements construct their own reality from their principles and program, and the resulting reality and discourses concern the set of everything that exists, from nature to technology including those strictly social elements.

This view of perspectivism, however, will constitute a limited novelty, already seen in other processes. It is important to add -what is fundamental, that which is its essence in the strategic sense of the word-that the construction of such ontological perspective - and no only cognitive-is based on "devouring" the other culture, and the other civilization, with which it is confronted.

Only then it will have a full sense of the diverse components of perspectivism that are analyzed here as structuring elements for social movements: performativity -that has been mentioned before-vincularity and aspectuality.

Forma sugerida de citar: $\quad$ Rojas Reyes, C. (2015). Perspectivismo indígena. Universitas, XIII (22), pp. 15-35. Quito: Editorial Abya-Yala/Universidad Politécnica Salesiana.

1 Filósofo, docente de la Universidad de Cuenca. 
Therefore, the central thesis is that when indigenous people live and narrate their experiences from their own perspective, they are not only representing themselves, but also effectively creating their cultures; they allow the emergence and the continuity of their ways of life. In this sense we move pass the thesis of Jose SanchezPraga, to move from recognition to the constitution of indigenous communities.

Keywords

Perspectivism, indigenous, performativity, vincularity, way of life.

\section{La necesidad de una ontología de los otros}

Hay una ontología, una epistemología, una ética, una estética en Occidente, constituidas de modo canónico, respecto de las cuales las posiciones diferentes son consideradas como desviaciones, como formas disminuidas, no siempre plenamente válidas. Todo lo que queda fuera tendría una ansiedad de llegar a ser como estos ideales. Nos hemos pasado preguntándonos si nuestra filosofía es filosofía en sentido estricto, si nuestro arte para ser tal tiene que cumplir con los parámetros del arte occidental, si nuestras democracias no son constantemente deficitarias contrastadas con los modelos externos de las sociedades desarrolladas, si nuestro capitalismo no es solo un espejismo del gran capital y, sobre todo, si la conformación de otros sujetos sociales tiene que seguir los mismos parámetros que los nuestros.

Entonces, cabe introducir la pregunta primera -en sentido ontológico, aunque no epistemológico- precisamente sobre el ser o con más precisión sobre el llegar a ser, ya que ser es llegar a ser. Hay una ontología. Pero, ¿hay una ontología de los otros?, ¿una ontología de los afroamericanos, de las mujeres, de los pobres, de los oprimidos, de los homosexuales, de los obreros, de los indígenas?

Esta es una pregunta que remite necesariamente a otra: ¿cuál es la forma de vida de los otros?, ¿cómo podemos acceder a ella?, ¿de qué modo podemos imaginarla no desde nosotros sino tal como ella misma se ve? (Spivak, 2012).

Hay una ética de los otros, como se puede ver en Dussel (2001). 
Hay una estética de los otros, Ticio Escobar (1993).

Hay una epistemología de los otros, López (1999).

¿En dónde estaría enunciada o anunciada una ontología, como forma de vida, de los otros? Ciertamente in nuce en todos estos desarrollos. Hace falta, sin embargo, una reflexión directa y estricta sobre la forma de vida de los otros.

Junto con esto hemos privilegiado aquellas ontologías que me parecieron que podían expresar de mejor manera la ontología de los otros, en sus necesidades y perspectivas contemporáneas. Dos vías se recorrieron: las ontologías sudamericanas de manera especial la ontología amazónica y bastante más complementaria la razón andina; y aquellas ontologías que significaban una ruptura con los cánones occidentales aun pensando dentro de Occidente, tales como (Negri y Hardt, 2002; Deleuze y Guattari, 2002; Agamben, 2008, 2014; Haraway, 2012).

Sin embargo, cuando pasamos de la ética o de cualquiera de las regiones señaladas a la epistemología, se produce un cambio de registro, una alteración radical en las consideraciones relativistas. Podemos afirmar sin demasiadas complicaciones que hay un canon occidental para el arte, o que tenemos diferentes éticas; pero esta aseveración no se sostiene para la ontología.

Ciertamente que es posible enunciar: hay una ontología occidental y una ontología de los otros. Este enunciado no se sostiene en cuanto a su verdad, esto es a su acuerdo con lo existente. Aquí hay una disyunción completamente excluyente: o lo uno o lo otro, sin posibilidad de término medio, de coexistencia ni conflictiva ni pacífica. Tenemos éticas contrapuestas que están en constante conflagración. En la ontología, hay una sola que es la mejor verdad posible en este momento dado; la otra necesariamente está equivocada, es falsa, no se corresponde con lo existente.

Quiero decir que la ontología huye del relativismo en la misma medida en que escapa del dogmatismo. Imposibilidad del relativismo porque hay una sola realidad y la representación que tengamos de ella solo puede ser una. No se puede decir sobre la realidad lo mismo y lo contrario al mismo tiempo bajo el mismo aspecto.

Imposibilidad del dogmatismo, porque la ontología nunca está completa; siempre es una aproximación a lo que realmente es. O, de otro modo, siempre habrá una teoría mejor que la que ahora tenemos.

La imagen que arroje la ontología de los otros será incomposible con la ontología actualmente dada en Occidente como canónica, en la medida en que remite a mundos incompatibles, porque enuncian sujetos, objetos, relaciones 
completamente diferentes e incompatibles, porque son formas de vida opuestas aunque interrelacionadas.

A fin de poder fundamentar adecuadamente lo que se entiende por ontología de los otros, es preciso confrontarse con algunos debates técnicos que son indispensables. Esto permitirá introducir una terminología técnica que deberá quedar debidamente clarificada.

La mirada ontológica que retomo aquí es el perspectivismo de Viveiros de Castro (2002) que lo aproximaré al ocasionalismo de Whitehead (1978) y a los planos de consistencia de Deleuze y Guattari (2002), para sostener que en esencia dicen lo mismo. Introduciré aquí las reflexiones de José Sánchez-Parga (2013) sobre la forma de vida indígena, aunque él usa otra terminología.

Postular una ontología fuera de los marcos tradicionales de la filosofía resulta particularmente difícil, porque las palabras apenas si alcanzan a decir lo que se trata de decir, porque aquello que se quiere expresar quizás ocupa el lugar provisional de lo inexpresable.

Constantemente estamos luchando con las palabras elegidas: perspectivismo, ocasionalismo, planos de consistencia, discordancia de los tiempos... todos dicen un aspecto de lo que se quiere enunciar, pero no existe aún un solo término que nos permita enunciar esta nueva ontología con la precisión y rigor que se quisiera. Una muestra de esta enorme dificultad puede verse en el sentido que le da Whitehead al término "feeling": como capacidad de prehensión del mundo que define cada elemento del universo, para expresar el núcleo básico de esa nueva ontología y con este nos toparemos más adelante (Whitehead, 1978).

Y no se trata únicamente de dificultades en el orden de la lengua, sino que la existencia apenas vislumbrada de otro mundo posible, el modo embrionario, frágil, de difícil reconocimiento, hace que la relación entre palabra y objeto se desquicie mucho más a menudo de lo que se quisiera y que aquellos significados que tratamos de evitar se cuelen por la ventana: relativismo, esencialismo, dogmatismo, dualismo.

\section{Dilucidación del perspectivismo ontológico desde la ontología amazónica}

Entremos ahora en una dilucidación del concepto de ontología y de sus elementos que nos permita una clarificación adecuada de la noción de perspecti- 
vismo y que justifique el que hablemos de perspectivismo ontológico, como un término técnico en el sentido en que quedará precisado más adelante.

Partiré de una afirmación ontológica básica que sustenta todo el edificio, que ya he citado: "ser es llegar a ser". La pregunta ontológica por el ser se resuelve completamente en esta otra: llegar a ser. Así que si queremos averiguar qué es algo, tenemos que indagar por los modos de su llegar a ser, por los caminos que tuvo que recorrer para desembocar en lo que es; esto es, esa precisa forma de vida.

Una primera imagen puede servir para aproximarnos al perspectivismo ontológico: los conos de la teoría de la relatividad, que explican la manera cómo cada segmento de la realidad está conformado exclusivamente por aquello que está al alcance de la velocidad de la luz. Hay que añadir a estos conos precisamente la idea de perspectiva, en el sentido de que cada uno de ellos es una determinada perspectiva del mundo.

Esta perspectiva les hace ser lo que son, proporcionándoles un modo específico de existencia concreta.

La primera y básica cuestión aquí radica en que la perspectiva es una característica ontológica y no se refiere a procesos cognoscitivos, peor aún a algún tipo de relativismo. Esto es, cada cono se proyecta y ocupa un segmento del espacio-tiempo. Esta proyección constituye por sí misma un acto creativo: ese segmento del universo comienza a existir en la medida en que el cono se proyecta; antes de eso no existe.

Veamos cómo esto se expresa desde la mirada de la ontología amazónica, que es de donde hemos tomado la idea principal: “...las cosas y los seres son los puntos de vista... La cuestión aquí, por tanto, no es saber cómo los monos ven el mundo..., sino qué mundo se expresa a través de los monos, de qué mundo son ellos un punto de vista" (Viveiros de Castro, 2002: 385).

Aquí hay una afirmación fundamental: las cosas son puntos de vista; no se trata de cómo los seres que nos representamos la realidad vemos el mundo de manera diversa, sino que el mundo se expresa de modo diverso a través de todo lo que existe.

La pregunta clave en todos los casos sobre qué es la realidad, de qué manera está constituida, cuál es su modo de existir, remite siempre a esta otra: de qué mundo son puntos de vista. Cada cono de lo real es un punto de vista, una perspectiva. Cada forma de vida es una perspectiva; no se dice que tiene una perspectiva, sino que es una perspectiva.

En su despliegue la totalidad de lo existente se expresa de diferentes modos; es una permanente creación de la diversidad. Aquí el énfasis lo ponemos en el 
término: modo. Cada cosa es una modalidad. Una vez que se ha constituido, que ha emergido como fruto de una concrescencia, ese modo prehende el mundo desde su propia perspectiva; y lo que es todavía mucho más importante, su ser no es otro que la forma en la que se da esa prehensión del mundo.

El perspectivismo implica, a su vez, como su consecuencia lógica que se producen constantemente realidades diversas, en cuanto cada una de ellas incoa que el mundo ha adquirido una determinada forma concreta, específica, en cierto modo irrepetible.

El mundo es un generador imparable de diversidades, es una máquina de distinciones, de segmentaciones interminables, que desembocan en una "economía general de la alteridad”: “...para vincular a la cuestión de puntos de vista no-humanos y de naturaleza relacional de las categorías cosmológicas un cuadro más amplio de manifestaciones de una economía general de la alteridad" (Viveiros de Castro, 2002: 352).

Cada elemento del mundo, sea humano o no, es un punto de vista diverso, una perspectiva que mira en cierta dirección, por medio de la cual el mundo atrapa ese segmento específico de lo real y al hacerlo, lo torna existente. Hay una relación directa entre perspectiva y llegar a ser.

El llegar a ser de las cosas -humanas y no humanas-, tiene la connotación de ser prehensiones de "puntos de vista distintos": “...trátase de una concepción, de muchos pueblos del continente, según la cual el mundo está habitado por diferentes especies de sujetos o personas, humanas y no-humanas, que aprenden según puntos de vista distintos" (Viveiros de Castro, 2002: 347).

Preguntarse por las características de una realidad, de cualquier tipo que estas sean, remiten constantemente a la capacidad de composición en la que ellas puedan entrar, desde su propia perspectiva, desde la que y a partir de la cual, son elementos diferenciables de los demás.

Sigo aquí la terminología de Whitehead que distingue entre prehensión y aprehensión, dejando esta segunda para el ámbito específicamente humano. Todos los entes a partir de su perspectiva prehenden el mundo y los seres humanos somos una expresión de esta prehensión y aprehendemos la realidad (Whitehead, 1978).

El perspectivismo se transforma inmediatamente en una forma de prehensión; o, si se prefiere, tener una perspectiva significa apropiarse del mundo de cierto modo; la perspectiva es siempre perspectiva de... Tiene un carácter intencional, como señalaremos más adelante. 
Pero, ¿qué es prehender? La prehensión es feeling, otra vez en el sentido de Whitehead: "Un afecto es la apropiación de algunos elementos en el universo para que sean componentes de la constitución real interna de este sujeto" (1978: 231).

Por tanto prehender el mundo siempre es un acto relacional, sin cuya relación no podría existir. Deviene actual exclusivamente en el momento en el que se apropia de determinados elementos para adquirir una cierta forma: "Prehensiones, o hechos relacionales concretos" (Whitehead, 1978: 22).

Junto con este carácter permanente de nexo, las prehensiones están determinadas, son determinaciones, máquinas de producir diversidad: "Un afecto es en todos sus aspectos determinado, con un sujeto determinado, unos datos iniciales determinados, unas prehensiones negativas determinadas, un dato objetivo determinado, y una determinada forma subjetiva" (Whitehead, 1978: 221).

Tener una perspectiva desde la cual se prehende el mundo remite al cómo ese sujeto prehende la realidad. Este cómo es tanto la razón de su existencia como el principio que le permite especificarse como distinto de todos los demás componentes del mundo: “...la forma subjetiva que es cómo este sujeto prehende este dato" (Whitehead, 1978: 23).

El viejo problema metafísico del ser se traslada a una cuestión bastante específica: cuál es su punto de vista, desde el que prehende el resto de la realidad. De allí que: “...una perspectividad - una capacidad de ocupar un punto de vista- es una cuestión de grado y de situación..." (Viveiros de Castro, 2002: 353).

Lo que implica una continuidad ontológica de todo el universo, desde los entes físicos hasta las realidades más espirituales, atravesando por los mundos virtuales. El conjunto completo de los componentes del mundo está formado por perspectivas que varían en alguna medida, que expresan situaciones de diversa índole. Así se trata de superar el dilema entre los aspectos universales y la existencia de la diversidad, en donde todo es perspectiva pero todo es perspectiva diversa.

Hay que insistir en la inversión que se ha provocado con este conjunto de afirmaciones.

Mas hay una cuestión más importante aquí. ¿Una teoría perspectivista amerindia está de hecho, como afirma Arhem, suponiendo una multiplicidad de representaciones sobre el mismo mundo? Basta considerar lo que dicen las etnografias, para percibir que es exactamente de modo inverso lo que se da; todos los seres 
ven (se representan) el mundo de una misma manera -lo que cambia es el mundo que ellos ven (Viveiros de Castro, 2002: 378).

Se trata de huir del relativismo que a primera vista parece saltar adelante, ante nuestros ojos. Oímos perspectiva y la asociamos inmediatamente con una propiedad de la mirada, como una cierta construcción virtual que nosotros colocamos sobre la realidad. Pero aquí el concepto de perspectiva es primero y primordial, anterior a su uso referido con la mirada o con cualquier otra situación específicamente humana.

Esta prehensión desde una perspectiva determinada tiene poco que ver, en este nivel, con cualquier tipo de representación o de conciencia. Tampoco se refiere a una suerte de relativismo generalizado. Lo que decimos con perspectivismo coincide plenamente con la teoría de la relatividad, en la medida en que se sostiene que las acciones de un objeto solo pueden influenciar sobre otro objeto si están dentro del mismo cono temporal; y por otra parte, que su movimiento solo puede ser calculado desde otro movimiento; en otros términos, que no existe ni el tiempo ni el espacio absolutos.

La perspectiva no solo está vinculada con la representación, peor con "una multiplicad de representaciones sobre el mismo mundo"; se refiere al hecho de que el mundo es el que varía constantemente y por esto hacemos el esfuerzo constante de representarlo de "la misma manera", a fin de poder aprehenderlo de alguna manera y de que no se nos escape.

Esta concepción se conoce con el nombre de multinaturalismo frente al multiculturalismo, en donde las aproximaciones cognoscitivas son las que tratan de mantenerse constantes frente a un mundo en perpetua variación:

El perspectivismo no es un relativismo, sino un multinaturalismo. El relativismo cultural, un multiculturalismo, supone una diversidad de representaciones subjetivas y parciales, incidentes sobre una naturaleza externa, una y total, indiferente a la representación; los amerindios proponen lo opuesto: una unidad representativa o fenomenológica puramente pronominal, aplicada indiferentemente sobre una diversidad real. Una sola cultura, múltiples naturalezas; epistemología constante, ontología variable -el perspectivismo es un multinaturalismo, pues una perspectiva no es una representación (Viveiros de Castro, 2002: 379).

La última frase de esta cita merece entonces ser resaltada una y otra vez para evitar equívocos: "una perspectiva no es una representación". El camino de las representaciones es, más bien, el de la epistemología que quiere con- 
vertirse en una constante, en un paradigma o programa de investigación, para estabilizar de alguna manera el mundo que, en cambio, está sometido a una ontología variable. En la hermosa frase de Viveiros de Castro: "Lejos del esencialismo espiritual del relativismo, el perspectivismo es un manierismo corporal" (2002: 380).

Tiene, desde luego, consecuencias importantes sobre los debates epistemológicos que no pueden ser reducidos a diversas aproximaciones sobre el mundo, cada una desde su relatividad, como era el caso de los paradigmas y de su inconmensurabilidad.

Detrás de las cuestiones epistemológicas yacen las preguntas ontológicas; subyacentes a las miradas epistemológicas están aquellas que remiten a esa variabilidad de formas de vida: ¿si estamos en desacuerdo con nuestras miradas sobre el mundo, no será que los mundos que habitamos son diversos?

Irreductibilidad de las diferencias a diferencias de opinión. Podría ser que nuestros desacuerdos remiten a que los mundos que habitamos, las perspectivas que son específicas y concretas, sean incompatibles con el mundo que otros habitan. Hay un choque de perspectivas, de segmentos del mundo que lo expresan de diversa manera. También en el campo de lo social habría que introducir junto al multiculturalismo el multinaturalismo. Más adelante veremos sus implicaciones.

En segundo lugar, y por fin: si los indios tienen razón, entonces la diferencia entre los dos puntos de vista no es una cuestión cultural, y mucho menos de mentalidad. Si el contraste entre relativismo y perspectivismo o entre multiculturalismo y multinaturalismo fueron leídos a la luz, no de nuestro relativismo multicultural, sino de una doctrina indígena, es forzoso concluir que la recíproca se aplica a la misma, y que la diferencia es de mundo, no de pensamiento... (Viveiros de Castro, 2002: 399).

O si se prefiere: las diferencias de pensamiento son, en último término, diferencias de mundo.

La noción de perspectiva tiene, además, otra cara; y por esto, hay que seguirla utilizando, a pesar de las connotaciones espontáneas que provoca. Este otro lado, se refiere a la relación que tiene con los sujetos.

En primer término la perspectiva hace relación con la intencionalidad: “... una teoría de que el universo está poblado de intencionalidades extra-humanas dotadas de perspectivas propias" (Viveiros de Castro, 2002: 357). 
Habría que darle a este aspecto un tratamiento husserliano, en el sentido de que la intencionalidad no es una características humana, sino que es previa a todo; es un dato de la realidad inmediata en todo sentido; así como toda conciencia es siempre conciencia de..., toda perspectiva se dirige, se orienta, se vuelca sobre y a esto le llamamos intencionalidad (Husserl, 1991).

El perspectivismo es integralmente relacional: "Tendríamos, con esto, una ontología integralmente relacional, en la cual las substancias individuales o las formas substanciales no son la realidad última" (Viveiros de Castro, 2002: 385).

Porque la realidad última, aquello que finalmente está detrás de todo como su origen, como su fundamento, es la perspectiva, el mundo como punto de vista y cada aspecto de este, como punto de vista diverso.

El perspectivismo intencional nos lleva de la mano a la noción de sujeto, diríamos que la exige como uno de sus postulados. Aquí hay, nuevamente, una inversión radical que es preciso resaltar con la mayor claridad posible.

No es el sujeto el que tiene una perspectiva sobre el mundo; es el mundo en cuanto perspectiva el que pone, coloca a partir de si el sujeto o, mejor aún, es sujeto en cuanto es perspectiva.

La posición de perspectiva conlleva la posición de sujeto: "el punto de vista crea el sujeto":

Todo ser al que se atribuye un punto de vista será entonces un sujeto, espíritu; o mejor, allí en donde estuviera un punto de vista, también estará una posición de sujeto. En cuanto a nuestra cosmología construccionista puede ser resumida en la fórmula saussureana: el punto de vista crea al objeto -el sujeto siendo la condición originaria de donde emana el punto de vista, el perspectivismo amerindio procedo según el principio de que el punto de vista crea el sujeto; será sujeto quien se encuentre activado o agenciado por el punto de vista (Viveiros de Castro, 2002: 373).

El perspectivismo es un proceso constante de subjetivación. Pero, ¿qué se quiere decir con esto? El sujeto en este contexto específico de la teoría del perspectivismo ontológico, significa la capacidad de prehender el mundo -y más delante de aprehenderlo-, dada su relacionalidad intrínseca:

El término sujeto se ha conservado a causa de que es familiar en filosofía. Pero me equivoco. El término superject sería mejor. El sujeto-superject es el propósito del proceso que origina el afecto. El afecto es inseparable del fin al cual tiende; y este fin es la afección (Whitehead, 1978: 222). 
El proceso por el cual una perspectiva prehende el mundo es un feeling y este a su vez constituye un sujeto. El sujeto es el lugar desde donde se mira el mundo de una determinada manera concreta. Este proceso tiene un valor ontológico y no se está refiriendo aquí a algún tipo de componente cognoscitivo o epistemológico, aunque ciertamente tendrá consecuencias de este tipo.

La perspectiva es siempre la perspectiva de... No cabe hablar de perspectiva sin más; es esta perspectiva que abre un "horizonte de sucesos", como dirá la teoría de la relatividad. Ser sujeto es, entonces, la capacidad de la realidad de definir los sucesos que son posibles dentro de un determinado cono de luz, o desde la perspectiva que expresa esa parcela del universo.

Se llega a través de estas consideraciones a una redefinición de las relaciones de naturaleza y cultura, que dejan de estar naturalizadas. Ahora ha sido el modelo social, humano, el que sirve para definir el mundo, porque hay una continuidad entre naturaleza y cultura que no puede romperse.

En términos de la ontología amazónica, a esto se le define como animismo: "El animismo puede ser definido como una ontología que postula el carácter social de las relaciones entre las series humana y no-humana: el intervalo entre naturaleza y sociedad es propiamente social" (Viveiros de Castro, 2002: 364).

Usamos las categorías sociales para definir ontológicamente el mundo:

Mas es realmente posible, y sobre todo interesante, definir el animismo como una proyección de las diferencias y cualidades internas al mundo humano sobre el mundo no-humano, esto es, como un modelo sociocéntrico en donde las categorías y relaciones intrahumanas son usadas para mapear el universo (Viveiros de Castro, 2002: 366).

Pero al mapear el universo desde lo sociocéntrico, lo humano tal como entendemos tradicionalmente, también se altera, quedan cuestionados sus límites; la humanidad se ha tornado problemática en su definición: "Si todos tienen alma, nadie es idéntico en sí mismo. Si todo puede ser humano, entonces nada es humano inequívocamente. Una humanidad de fondo torna problemática la humanidad de forma" (Viveiros de Castro, 2002: 377).

La distinción entre animales y seres humanos tiene que rehacerse constantemente, debe a cada instante probarse, ya no está dada naturalmente: "Pues si, como sugerí, una condición común a los humanos y animales es una humanidad, no una animalidad, es porque humanidad es el nombre de la forma real del Sujeto" (Viveiros de Castro, 2002: 374). 


\section{El carácter ontológico de la performatividad}

Desde su origen lingüístico la performatividad pasa a las ciencias sociales, como noción útil para comprender el surgimiento de diversos fenómenos, tales como la constitución del género o los aspectos de la teoría queer.

En este parte se trata de mostrar la relación existente entre ontología y performatividad, que sostiene que el perspectivismo ontológico como prehensión de un sujeto -feeling- tiene un carácter performativo.

Una doble consecuencia se deriva de este tratamiento: primero sobre la ontología quitándolo cualquier rasgo de esencialismo o substancialismo; segundo, sobre la performatividad mostrando que no solo es un fenómeno lingüístico, sino que es tal en la medida en que tiene un valor ontológico.

Uno de los núcleos de esta ontología está en su capacidad des-esencializante, que además provee a todo lo existente de un estatuto inmanente. Nada está fuera, todo está dentro; no existe exterioridad posible.

Recordemos aquí lo que se entiende por performativo: Performativos: actos que producen la realidad que describen.

Y ahora produzcamos la transformación ontológica de esta proposición:

Perspectivismo performático o realizativo: actualizaciones de lo real que se producen desde el surgimiento de una perspectiva. Nada hay previo en lo real que no sea perspectiva, excepto aquellos fenómenos que se derivan de otras perspectivas, de sus cruces, de la formación de planos de consistencia. El origen y fundamento de lo actual es la perspectiva, que en cuanto prehende constituye aquello que prehende. Prehender significa crear lo que se prehende.

Por esto se puede establecer que: Perspectivismo: actos que producen la realidad que prehenden.

No tiene otro fundamento $\mathrm{u}$ origen que no sea el propio establecimiento de la una perspectiva determinada; lo que disuelve el esencialismo y tendrá consecuencias epistemológicas porque obliga al conocimiento a detenerse en el plano de la inmanencia. Y a su vez esto regresa sobre el perfomativo, para mostrar tal como dice la palabra española: realizativo.

Añadamos a esta primera aproximación de la ontología de los otros, una característica que le es fundamental: la vincularidad, que conduce a la inmanencia de los otros. 


\section{Vincularidad}

Elijo esta palabra para expresar diversas nociones que están contenidas dentro de ella y porque proviene del mundo andino: “...en el mundo andino... para que algo funcione, necesita su par o complemento, sino no sirve de mucho; y luego, como se puede colegir fácilmente, este complemento necesita ser proporcional al primer elemento con el que se encuentra vinculado" (Lajo, 2003: 95).

El énfasis primero hay que colocarlo en la necesidad del otro para existir y solo a partir de esta afirmación puede darse la estructura de la complementariedad. En el principio era el vínculo y era este el fundamento de la existencia. El vínculo como ontológico:

Es decir, bien y realidad, así como hombre y naturaleza en el mundo andino no son conceptos separados, sino están juntos en vínculo o yanantinkuy; el bien como el hombre se realizan, o fluyen con la realidad y la naturaleza, es más, estos representan la única forma para que el bien y el hombre puedan viabilizarse, existir o ser. Este contenido de sabiduría puquina se expresa con el término qhapaq (Lajo, 2003:112).

Citemos esos conceptos que incluimos bajo el término vincularidad: Relatedness, nexus, togetherness (Whitehead); Relacionalidad (Viveiros de Castro); Reciprocidad (Estermann); Devenir (Deleuze).

El Diccionario de la Real Academia de la Lengua Española define vincular como: "Vincular: tr. Atar o fundar algo en otra cosa... Someter la suerte o el comportamiento de alguien o de algo a los de otra persona o cosa".

Así se da un paso más delante de los conceptos de relación o nexo, porque introduce en ellos la inmanencia, ya que se funda algo en otra cosa. Decimos que ese vínculo es inmanente, que lo vinculado es inmanente; que en la constitución de lo real, la perspectiva del otro es inmanente al fenómeno en cuestión, aunque no fuera más que como la perspectiva diversa que permite que esta exista como tal.

Los quichuas extienden este principio a todo el cosmos: "En este contexto el principio de relacionalidad se manifiesta como la red de nexos y vínculos, que es la fuerza vital de todo lo que existe. Para este tipo de filosofía no existen seres absolutos, en el sentido tradicional del término. Hasta Dios es un ente relacionado" (Yánez, 2002: 38). 
En las nociones de la ontología amazónica, hay una inmanencia del enemigo, que se puede convertir en esta ontología general, en la inmanencia del otro. Veamos cómo.

Hay que ubicarse en la "economía de la alteridad", para ver cómo esta cambia desde esas miradas trascendentalistas de lo otro que lo colocan inevitablemente fuera, en una especie de exterioridad pura, hasta desembocar en una alteridad inmanente: “...cuidamos de que su examen pueda contribuir a un mejor entendimiento de un régimen simbólico de amplia difusión en la Amazonía indígena, una economía de la alteridad en donde al concepto de enemigo se le asigna un valor cardinal" (Viveiros de Castro, 2002: 267).

Cada componente del mundo es coextensivo en principio con el universo entero, pero lo es de hecho con un segmento definido de este. En los términos de la ontología amazónica, en el momento de capturar y luego devorar al enemigo, se pasa de la oposición completa a la identificación: "El enemigo se dice que está enfurecido como su matador, más al mismo tiempo se halla indisolublemente ligado a él... Ellos van de la alteridad mortífera a la identidad fusional" (Viveiros de Castro, 2002: 273).

El plano simbólico de la comunidad se altera para dar cabida a los significados que provienen del enemigo; los límites del mundo de la tribu se amplían en la medida en que los límites de su lenguaje crecen y se modifican para adquirir otras palabras o cambiar las existentes: "Vistos por su lado bueno -su lado muerto-, los enemigos son aquellos que traen nuevas palabras al grupo, o al menos que vienen a dar un sentido más puro a las palabras de la tribu (sic)" (Viveiros de Castro, 2002: 275).

Devorando al enemigo, las palabras de este te escogen, comienzas a hablar con las palabras del otro, hasta tener la capacidad de verte a ti mismo como otro, cambiando de perspectiva: "Esa capacidad de verse como Otro -punto de vista que es, talvez, el ángulo ideal de visión de sí mismo- me parece la clave de la antropología tupí-guaraní." (Viveiros de Castro, 2002: 281).

Y a continuación: "Se considera que la dinámica identitaria del par matador-víctima es un proceso de ocupación del punto de vista del enemigo" (Viveiros de Castro, 2002: 291).

$\mathrm{Y}$ este repercute en una transformación de las subjetividades, en cuanto estas derivan de la perspectiva adoptada. Sujeto es tener un punto de vista; estrictamente hablando, aunque en español suene raro: el sujeto es un punto de vista, una perspectiva; y viceversa: una perspectiva es el proceso de surgimiento de un sujeto. 
Pero de un sujeto enteramente relacional, en un proceso de digestión del otro a través de su prehensión perspectiva:

Si es verdad que el punto de vista crea el objeto, no es menos verdad que el punto de vista crea el sujeto, pues la función del sujeto se define precisamente por la facultad de ocupar un punto de vista. En este sentido, una asimilación predatoria de las propiedades de la víctima, en el caso amazónico, debe ser comprendida no tanto en términos de una física de las substancias como en los de una geometría de las relaciones, esto es, en cuanto movimiento de prensión perspectiva, en donde las transformaciones resultantes de agresión guerrera inciden sobre determinadas posiciones como puntos de vista (Viveiros de Castro, 2002: 291).

Por esto se produce una "fusão dos pontos de vista" y la relación de los predatores se convierte en proceso de subjetivación:

Una relación es creada precisamente por la supresión de uno de sus términos, que es introyectado por el otro; una dependencia recíproca que liga y constituye los sujetos del intercambio a que sea su punto de fusión-, en donde una distancia extensiva e intrínseca entre las partes se convierte en una diferencia intensiva, inmanente a una singularidad dividida. Una relación de predación se constituye en un modo de subjetivación (Viveiros de Castro, 2002: 293).

De la noción de afín, que será tratada más adelante, aparece aquí como crucial: "El Otro, en suma, es primero un Afín" (Viveiros de Castro, 2002: 416).

Con estos dos términos: performativo y vincularidad, podemos acercarnos de mejor manera a la cuestión de otras formas de vida, otras ontologías, especialmente aquellas que nos conciernen, las indígenas, bien sean andinas o amazónicas.

Señalemos que hay un vínculo inseparable de esas formas de vida con su forma; esto es, con su cultura, con la experiencia que cada indígena hace de ella, que impide que sea aprehendida fuera de este marco de referencia: "Con el término forma-de-vida, entendemos en cambio una vida que no puede más ser separada de su forma, una vida en la cual no es más posible aislar y mantener aislada cualquier cosa como una nuda vida" (Agamben, 2014: 264).

En la experiencia de una comunidad andina o amazónica siempre está presente el elemento común, la comunidad y el conjunto de posibilidades de expresión concreta que se derivan de esta. Fuera de su vínculo con la comunidad no alcanzarían a ser, se disolverían en otra forma de vida: 
La experiencia del pensamiento, que está aquí en cuestión, es siempre experiencia de una potencia y de un uso común. Comunidad y potencia se identifican sin residuo, porque la inherencia de un principio comunitario en cada potencia es función del carácter necesariamente potencial de cada comunidad (Agamben, 2014: 269).

En este momento es preciso ir más allá de los temas del reconocimiento por parte del otro, externo a la comunidad, extendiendo el tema de la experiencia que no solo es el modo en el que se vive la cultura sino que tiene un carácter performativo.

Hay que dar un paso delante de las afirmaciones de Sánchez-Parga para mostrar que la experiencia y sus representaciones que hace el indígena no solo son parte de un reconocimiento, sino que funcionan de modo performativo:

Una cosa es investigar y saber qué es un indígena a partir de los procesos de 'descomunalización' de la comuna andina y de individualización de la población indígena, así como a partir del cambio lingüístico cultural, y otra cosa muy diferente es la experiencia que él tiene de dichos procesos, cómo se los representa y se relaciona con ellos, y cómo a partir de estos sigue reconociéndose (Sánchez-Parga, 2013: 11).

Aquí lo que sostenemos es que la experiencia es experiencia de la cultura y al mismo tiempo el origen - performático- de dicha cultura. Más aún, cuando el indígena habla no solo enuncia su versión de su cultura, sino que en este proceso, la está creando:

De lo que se trataba en realidad era de transformar la pregunta en una escucha, no obligando al indígena a existir como tal en el discurso del antropólogo. Cuando este obliga a aquel a hablar sobre su cultura, como algo diferente de lo que él mismo es, este la objetiva y da una versión ideológica de ella; por el contrario, cuando el indígena habla, comunica ya una versión subjetiva de su cultura, siendo esta la que habla a través de todo lo que él dice (Sánchez-Parga, 2013: 103).

No es solo su "versión ideológica", sino su constitución que proviene de sus acciones, pero con igual fuerza de sus discursos, especialmente cuando lo hace frente al otro. Para hablar con el otro, constituye su identidad performaticamente; esto es, aquello que dice comienza a existir: "En conclusión el indígena lo es al interior de una diferencia y relación con "otro"; es respecto de un interlo- 
cutor que se reconoce como indígena, hablando con él, para ser reconocido en cuanto tal" (Sánchez-Parga, 2013: 104).

Cuando se le pregunta al indígena ¿qué es ser indígena para el indígena?, se obtiene ante todo una narración, no tanto una suerte de teorización. Pero esta narración si bien es un modo de representación de lo que vive y una exigencia de reconocimiento, es de manera preeminente una narración que crea aquello que se encuentra narrando:

La condición e identidad indígena no solo se hereda, se recibe, se transmite, sino sobre todo es algo que se hace e incesantemente se reconstruye. Cuando el indígena habla de sí mismo, se cuenta. El indígena no dice lo que es, sino más bien se narra. Cuanto él cuenta su vida y relata está diciendo lo que significó y significa para él ser indígena (Sánchez-Parga, 2013: 124).

Por eso se rebasa el "reconocimiento de su condición de sujetos" y se sostiene que se trata de su emergencia ontológica como tales, que reafirma su forma de vida y la permite persistir en el tiempo, aun a través de los procesos de mestizaje:

En conclusión, el actual proceso de mestizaje de gran parte de las poblaciones indígenas de ninguna manera comporta o acarrea necesariamente un proceso de desindigenización... De la misma manera que incluso los indígenas se encuentran relativamente marginales a la sociedad y cultura mestizas pueden reconocer el mestizaje de su identidad cultural, Lo cual significa que no hay identificación que no sea intercultural.

\section{Ontología ocasionalista, ontología aspectual}

La serie de elementos de la ontología de los otros que hemos tratado en esta sección pueden sintetizarse en la ontología ocasionalista/aspectual, que son dos nombres para expresar la misma situación, pero que proveen de matices indispensables para su comprensión adecuada.

Whitehead deriva de la mecánica cuántica esta característica general del mundo: todo es ocasión, como fenómeno originario y fundamentante; o, si se prefiere, solo existen ocasiones; lo demás son formaciones derivadas de esta, incluso aquello que nos parece estable, permanente, eterno. Las ocasiones son las que conforman planos de consistencia. 
Quizás el término en español no logra expresar plenamente lo que se quiere, aunque en su uso está contenido de manera implícito el sentido que le queremos: su radical contingencia. Cuando decimos "esta es una buena ocasión, aprovecha la ocasión, es una ocasión para...", nos referimos a un momento circunstancial, pasajero, que puede volver a presentarse o no y que exige una acción.

Whitehead desde luego lo usa en el sentido del inglés: "An occasion is an event or such a juncture of events as seems to require action."

La realidad última, ontológica, es ocasión: "Las entidades actuales -también llamadas ocasiones actuales- son la cosa real final de las el mundo está hecho. Nada hay detrás de las entidades actuales que sean más reales" (Whitehead, 1978: 18).

Esa contingencia radical del evento que requiere de una acción tiene que ser completado en su sentido de otros matices que den cuenta del perspectivismo ontológico, relacional, inmanente, que contiene al otro de manera inmanente y que desde su punto de vista prehende el mundo.

La noción de ontología aspectual pretende cubrir de manera sintética el conjunto de estas características.

Parafraseamos a Whitehead de la siguiente manera: Las entidades aspectuales -también llamadas entidades actuales u ocasionales-son las cosas reales últimas de las que el mundo está hecho. No hay cosa alguna detrás de las entidades aspectuales que nos permitan hallar algo más real.

El primer elemento que ya justifica su elección para relacionarlo con la ontología, es la introducción del tiempo en estas consideraciones, pero no se trata del tiempo estructurado en una secuencia de pasado, presente y futuro, de alguna forma gramaticalizado.

El aspecto se refiere al modo cómo pasa el tiempo concreta y efectivamente en una ocasión determinada; hace referencia, por ejemplo, a su duración; y, desde luego, se puede durar de muchas maneras muy complejas y sofisticadas. Lo que nos permitirá decir más adelante que la diversidad de perspectivas está vinculada a una diversidad de temporalidades, de modos de pasar el tiempo.

Hay una distinción clave entre tiempo y aspecto, porque el primero segmenta el continuo para mirarlo desde otro acontecimiento o desde la posición del que habla y el segundo representa el fluir del tiempo en una máxima diversidad. Todo fluye pero lo hace de modo diverso. En la gramática, precisamente se distingue entre el carácter deíctico del tiempo y el tiempo interno del evento.

El tiempo, en cuanto cronológico, se pone como universal, como un medio en el que todos los fenómenos suceden, desde donde se colocan las fechas: 
La estrecha relación existente entre el tiempo y el aspecto es consecuencia del hecho de que ambas nociones tienen que ver con la temporalidad de los eventos verbales, si bien otorgan a esta un tratamiento diferente. En efecto, el tiempo es una categoría deíctica: localiza el evento verbal en un tiempo externo, orientándolo en relación con el momento de habla, bien en relación con el tiempo en que tiene lugar otro evento (De Miguel, 1999: 2989).

Es de lo más interesante que el propio idioma reconozca este otro componente de los verbos, que es el aspecto; se suele decir que el aspecto es primero en el orden de aparición histórica que el tiempo, porque nace de la vida misma, de los hechos en cuanto sociales, subjetivos, teñidos por la realidad en su inmediatez: "El aspecto, en cambio, se ocupa del tiempo como una propiedad inherente o interna del propio evento: muestra el evento tal y como este se desarrolla o distribuye en el tiempo, sin hacer referencia al momento del habla" (De Miguel, 1999: 2989).

La independencia respecto del momento del habla le permite al aspecto tener un carácter ontológico general y referirse a todo lo que existe en cuanto lo hace a partir del tiempo que le es inherente. Así, el aspecto se convierte en el fenómeno fundamental de la lengua, porque corresponde a la descripción básica de cómo es el mundo, que proviene de la manera cómo ha llegado a ser, cuál es su tiempo interno: “... el aspecto no solo existe como categoría universal sino que es ontogénicamente anterior al tiempo, de manera que el niño que aprende una lengua con ambas categorías, adquiere antes el aspecto" (De Miguel, 1999: 2990).

Ahora se puede establecer que aquello que le dota de perspectiva a cada elemento del mundo, que le hace ser lo que es, proviene del modo cómo pasa el tiempo, cómo está estructurado su tiempo interno. El aspecto es el que el dota provee la perspectiva, aunque en realidad son fases inseparables: aspecto perspectivista o perspectivismo aspectual.

Sobre esta base devenimos lo que tengamos que devenir: devenir-animal, devenir-molécula, devenir-mujer, devenir-obrero, devenir-afroamericano..., que significa de manera primordial que ser (y por ende tener) una perspectiva aspectual, una proyección en el tiempo que lo atraviesa de una manera determinada (aspecto) y que le hace ser lo que es (Deleuze y Guattari, 2002).

Fuera de esta perspectiva aspectual nada se puede ubicar, no hay exterioridad posible. Pero esta perspectiva aspectual contiene el fragmento del mundo al que alcanza a mirar desde su perspectiva, de manera inmanente: 
En el devenir-mujer, el patriarcado en inmanente; no se puede devenirobrero sin el capitalista, ni devenir-afroamericano sin los hombres blancos, occidentales. En estos casos, literalmente el enemigo es inmanente.

De allí proviene su relacionalidad, su vincularidad radical, en donde miramos la diversidad como el núcleo de cualquier ontología: "La primera y principal de estas premisas es: una identidad es un caso particular de la diferencia... $\mathrm{O}$ lo que equivale a decir que solo existe la diferencia, en mayor o menor intensidad: esa es la naturaleza del valor medido" (Viveiros de Castro, 2002: 422).

\section{Bibliografía}

Agamben, Giorgio

2008 El reino y la gloria. Buenos Aires: Adriana Hidalgo.

2014 L'uso dei corpi. Vincenza: Neri Pozza.

De Miguel, Elena

1999 El aspecto léxico. Madrid: Espasa.

Deleuze y Guattari

2002 Mil mesetas. Valencia: Pre-Textos.

Dussel, Enrique

2001 Hacia una filosofía política crítica. Bilbao: Desclée de Brower.

Escobar, Ticio

1993 La belleza de los otros. Asunción: RP Ediciones.

Haraway, Donna

2012 "Manifiesto cyborg". 20 de diciembre de 2012. http://manifiestocyborg. blogspot.com/.

Husserl, Edmund

1991 La crisis de las ciencias europeas y la fenomenología trascendental. Madrid: Crítica.

Lajo, Javier

2003 Qhapaq ñan. La ruta inca de la sabiduría. Quito: Abya-Yala.

López, Carlos

1999 Los Popol Wuj y sus epistemologías. Quito: Abya-Yala.

Negri, Antonio y Hardt, Michael

2002 Imperio. Barcelona: Paidós Ibérica.

Sánchez-Parga, José

2013 ¿Qué significa para el indígena ser indígena? Quito: Abya-Yala. 
Spivak, Gayatri Chakravorty

2012 An aesthetic education in the era of globalization. Cambridge, MA.: Harvard University Press.

Viveiros de Castro, Eduardo

2002 A inconstancia da alma selvagem. Sao Paulo: Cosac \& Naify.

Whitehead, Alfred N.

1978 Process and reality. New York: The Free Press.

Yánez, José

2002 Yanantin. La filosofia intercultural del Manuscrito Haurochiri. Quito: AbyaYala.

Fecha de recepción: 17/3/2015; fecha de aprobación: 8/ 6/2015 